\title{
Strategy for successful expression of the Pseudomonas putida nitrile hydratase activator P14K in Escherichia coli
}

$\mathrm{Yi} \mathrm{Liu}^{1+}$, Wenjing Cui ${ }^{1 \dagger}$, Yueqin Fang ${ }^{2}$, Yuechun Yu ${ }^{1}$, Youtian Cui ${ }^{1}$, Yuanyuan Xia ${ }^{1}$, Michihiko Kobayashi ${ }^{3^{*}}$ and Zhemin Zhou ${ }^{1 *}$

\begin{abstract}
Background: Activators of Nitrile hydratase (NHase) are essential for functional NHase biosynthesis. However, the activator P14K in P. putida is difficult to heterogeneously express, which retards the clarification of the mechanism of P14K involved in the maturation of NHase. Although a strep tag containing P14K (strep-P14K) was overexpressed, its low expression level and low stability affect the further analysis.

Results: We successfully expressed P14K through genetic modifications according to N-end rule and analyzed the mechanism for its difficult expression. We found that mutation of the second N-terminal amino-acid of the protein from lysine to alanine or truncating the N-terminal 16 amino-acid sequence resulted in successful expression of P14K. Moreover, fusion of a pelB leader and strep tag together (pelB-strep-P14K) at the N-terminus increased P14K expression. In addition, the pelB-strep-P14K was more stable than the strep-P14K.
\end{abstract}

Conclusions: Our results are not only useful for clarification of the role of P14K involved in the NHase maturation, but also helpful for heterologous expression of other difficult expression proteins.

Keywords: NHase, N-end rule, Pseudomonas putida, P14K, Stability

\section{Background}

Nitrile hydratase (NHase, EC 4.2.1.84) is composed of $\alpha$ and $\beta$-subunits. The enzyme contains either a non-heme iron (Fe-NHase) [1] or non-corrin cobalt ion (Co-NHase) [2] in the active center and catalyzes the hydration of a nitrile to the corresponding amide, which is followed by several consecutive reactions: amide $\rightarrow$ acid $\rightarrow$ acyl-CoA, as catalyzed by amidase [3] and acyl-CoA synthetase [1], respectively. The metal ions in both Co-NHase and FeNHase are located in their $\alpha$-subunits, which share a characteristic metal-binding motif [CXLC $\left.\left(\mathrm{SO}_{2} \mathrm{H}\right) \mathrm{SC}(\mathrm{SOH})\right]$ containing two modified cysteine residues: cysteine-sulfinic acid $\left(\alpha \mathrm{Cys}-\mathrm{SO}_{2} \mathrm{H}\right)$ and cysteine-sulfenic acid $(\alpha \mathrm{Cys}-\mathrm{SOH})$

\footnotetext{
*Correspondence: kobay@agbi.tsukuba.ac.jp; zhmzhou@jiangnan.edu.cn ${ }^{\dagger}$ Equal contributors

${ }^{3}$ Institute of Applied Biochemistry, and Graduate School of Life and Environmental Sciences, The University of Tsukuba, 1-1-1 Tennodai, Tsukuba, Ibaraki 305-8572, Japan

'Key Laboratory of Industrial Biotechnology, Ministry of Education, School of Biotechnology, Jiangnan University, Wuxi 214122, Peoples Republic of China Full list of author information is available at the end of the article
}

$[1,4,5]$. The apoenzyme is likely to be unmodified, according to previous studies on NHase [6] and a related enzyme, thiocyanate hydrolase (SCNase) [7].

The trafficking of metal ions into NHases is mediated by various "activator proteins" [8]. Fe-NHases require activators for functional expression in Rhodococcus sp. N-771 [9], Pseudomonas chlororaphis B23 [10] and Rhodococcus sp. N-774 [11]. A proposed metal-binding motif, CXCC, in the NHase activator of Rhodococcus sp. N-771 has been identified and the activators for Fe-type NHases have been shown to act as metallochaperones [12]. For the two Co-NHases (L-NHase and H-NHase) in Rhodococcus rhodochrous J1, cobalt incorporation has been found to be dependent on self-subunit swapping: the activator protein exists as a complex with the $\alpha$-subunit of NHase, the cobalt incorporation involves the swapping of the cobalt-free $\alpha$-subunit of the cobaltfree NHase with the cobalt-containing $\alpha$-subunit of the complex [13-15]. NHase in Pseudomonas putida NRRL18668 and acetonitrile hydratase (ANHase, an NHase

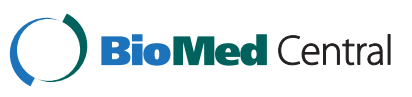


that catalyzes the hydration of small aliphatic nitriles) from Rhodococcus jostii RHA1 are also Co-NHases, in which P14K and AnhE, respectively, are essential for NHase maturation $[16,17]$. However, their gene organizations are quite different from those of L-NHase and $\mathrm{H}$ NHase. The structural genes of L-NHase and H-NHase have the order $<\beta$-subunit $><\alpha$-subunit $><$ self-subunit swapping chaperone $>$, while those in ANHase and the NHase of $P$. putida NRRL-18668 have the order $<\alpha$-subunit $><$ AnhE $><\beta$-subunit $>[16]$ and $<\alpha$-subunit $><\beta$-subunit $><\mathrm{P} 14 \mathrm{~K}\rangle$ [17], respectively, with the latter protein being identical to the metallochaperone in Fe-NHase except that the molecular mass of the protein in Fe-NHase is larger than P14K. While AnhE has been found to act as a metallochaperone (not as a self-subunit swapping chaperone) during cobalt incorporation into ANHase [16], very recently, we discovered that cobalt incorporation into the NHase of $P$. putida NRRL-18668 is also dependent on the self-subunit swapping, and the P14K is a complex with the $\alpha$-subunit [18]. However, the P14K is difficult to be heterogeneously expressed, though a strep tag containing P14K was expressed, its low expression level and low stability retard the further clarification of their detailed role for cobalt incorporation.

Heterologous expression systems are commonly used for protein research. Protein degradation in heterologous expression systems often leads to failure for the isolation of proteins of interest. Intracellular protein degradation plays an essential role in many physiological processes by removing damaged polypeptides and proteins that harbor specific destruction tags. N-end pathway degradation relates the metabolic stability of a protein to the N-terminal residue of that protein [19]. The $\mathrm{N}$-end rule defines the stability of proteins according to the nature of their $\mathrm{N}$ terminal residues. These residues are classified as stabilizing and destabilizing residues, which serve as recognition determinants for protein degradation [19-21]. In bacteria such as E. coli, the N-end rule pathway is present. According to the $\mathrm{N}$-end rule, amino-terminal arginine, lysine, leucine, phenylalanine, tyrosine and tryptophan confer 2 minute half-lives to proteins, while the other amino-terminal residues confer greater than 10 hour halflives to the same proteins (Figure 1) [22,23].

In the present study, we successfully expressed the NHase activator P14K through site-specific mutagenesis taking into account $\mathrm{N}$-end rule degradation. We also increased the expression and the stability of P14K by fusion of a pelB leader and strep tag together at its N-terminus. These results are useful for elucidation of the mechanism of cobalt incorporation into the $\alpha$-subunit of NHase in P. putida NRRL-18668. Furthermore, these strategies, which promote the over-expression of the instable P14K in $E$. coli, might also be helpful for the heterologous expression of other difficult expression proteins.

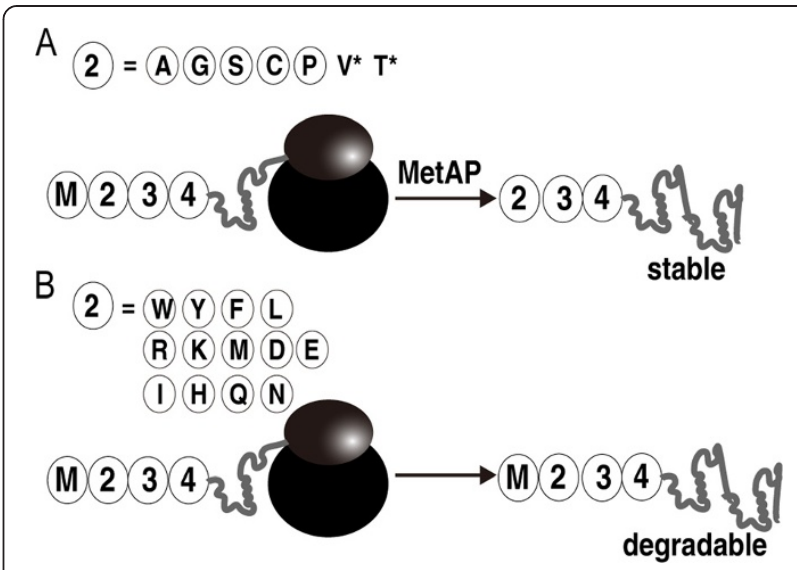

Figure 1 The schematic diagram of $\mathrm{N}$-end rule in bacteria. (A) Relatively long protein half-life. If the second $\mathrm{N}$-terminal amino-acid is $A, G, S, C$ or $P$ and in some cases $V$ or $T$, which can be processed by methionine aminopeptidase (MetAP), the protein will have a long half-life. (B) Relatively short protein half-life. When the second Nterminal amino-acid is $W, Y, F, L, R, K, M, D, E, I, H, Q$ or $N$, which can not be processed by MetAP, the protein will have a short half-life.

\section{Results and discussion}

Molecular modification to improve the yield of P14K from P. putida NRRL-18668

To clarify the mechanism of cobalt incorporation into the NHase of P. putida NRRL-18668, we attempted to isolate $\mathrm{P} 14 \mathrm{~K}$, which is essential for NHase activity [17]. The transformant harboring $\mathrm{pET}-A B P$, which contained the NHase and P14K genes, was used for NHase and P14K expression. As a result, although NHase was successfully expressed, P14K was hardly detected on SDS-PAGE. This result is consistent with the previous report [17]. Although a strep tag containing P14K was expressed using a transformant harboring pET- $A B($ strep- $P$ ), and the strep-P14K was found to be a complex with the $\alpha$-subunit $[\alpha$-(strep-P14K)] [18], the low expression level and the low stability of the complex retard the further clarification of its detailed role for cobalt incorporation, a strategy for enhancing P14K expression level and stability is expected. It has been shown that heterologous expression of proteins in E. coli can be enhanced by using a synthetically strong ribosome binding site (RBS) [24], reducing the distance between the promoter region and target gene [25] and gene codon optimization [26]. To enhance the expression of P14K, the putative RBS (CTGGAG) within the $B$ gene (encoding the $\beta$ subunit) (Figure 2A) was replaced with an enhanced RBS (AAGGAG) between gene $B$ and gene $P$ (encoding $\mathrm{P} 14 \mathrm{~K})$ during the construction of the plasmid $\mathrm{pET}-A B^{\prime} P$ (Figure 3). Simultaneously, the plasmid pET-PAB, with the gene order $\langle P\rangle\langle A\rangle\langle B\rangle$, was constructed. In this plasmid, the distance between the promoter sequence and the P14K gene was shortened and the strong RBS was inserted upstream of each gene (Figure 3). 


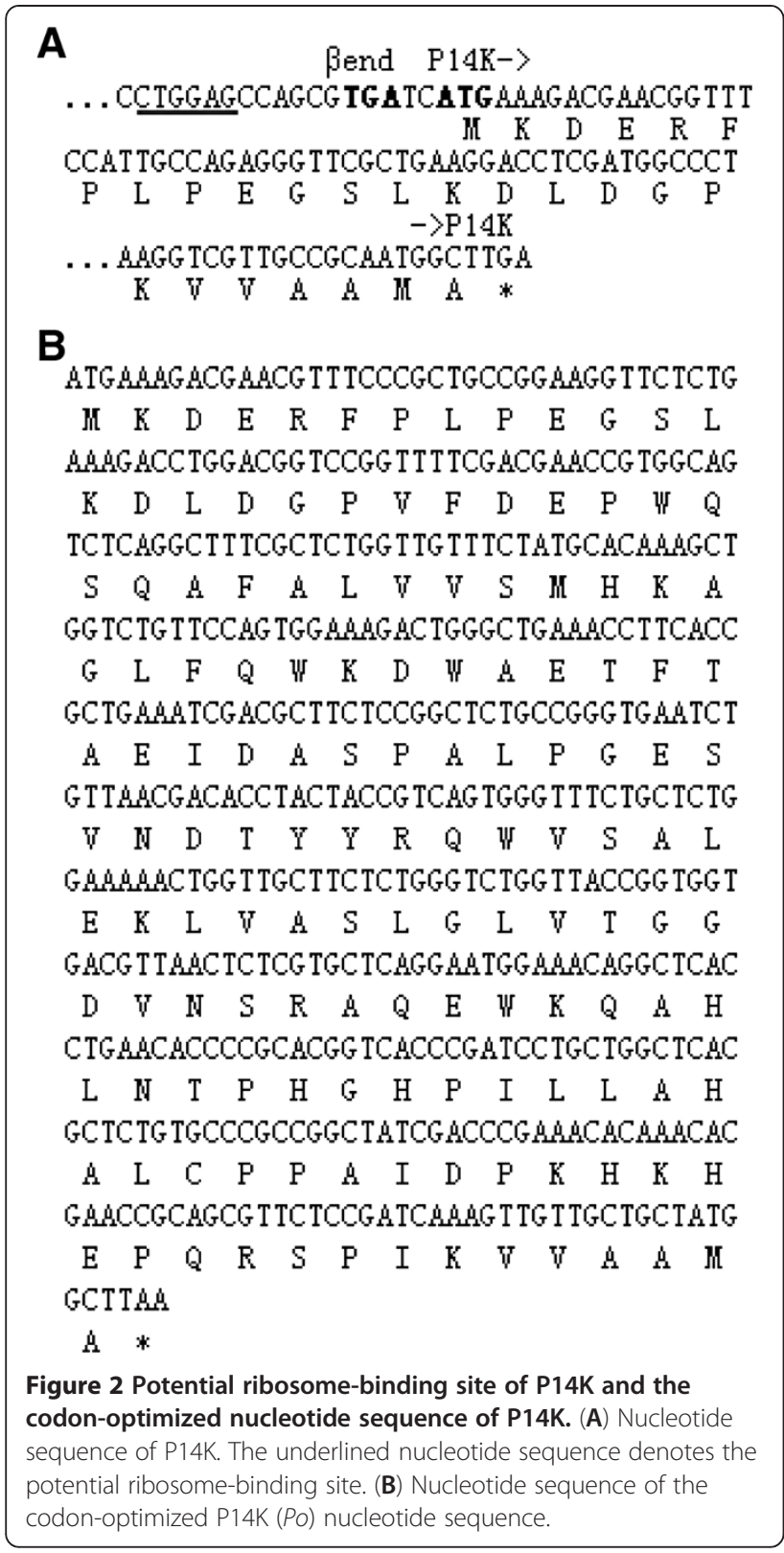

Moreover, plasmid pET-ABPo, with optimized P14K gene codons and a strong RBS for each gene, was also constructed (Figure 2B). However, P14K was not abundantly expressed by the transformants harboring $\mathrm{pET}-A B^{\prime} P$, pET-PAB or pET-ABPo (Figure $4 \mathrm{~A}$ ).

\section{Successful heterologous expression of P14K}

The N-end rule states that the half-life of a protein is determined by the nature of its N-terminal residue [19-21]. This fundamental principle of proteolytic regulation is conserved from bacteria to mammals [19]. N-terminal arginine, lysine, leucine, phenylalanine, tyrosine and tryptophan confer $2 \mathrm{mi}-$ nute half-lives to proteins, while the other $\mathrm{N}$-terminal residues confer greater than 10 hour half-lives to the same

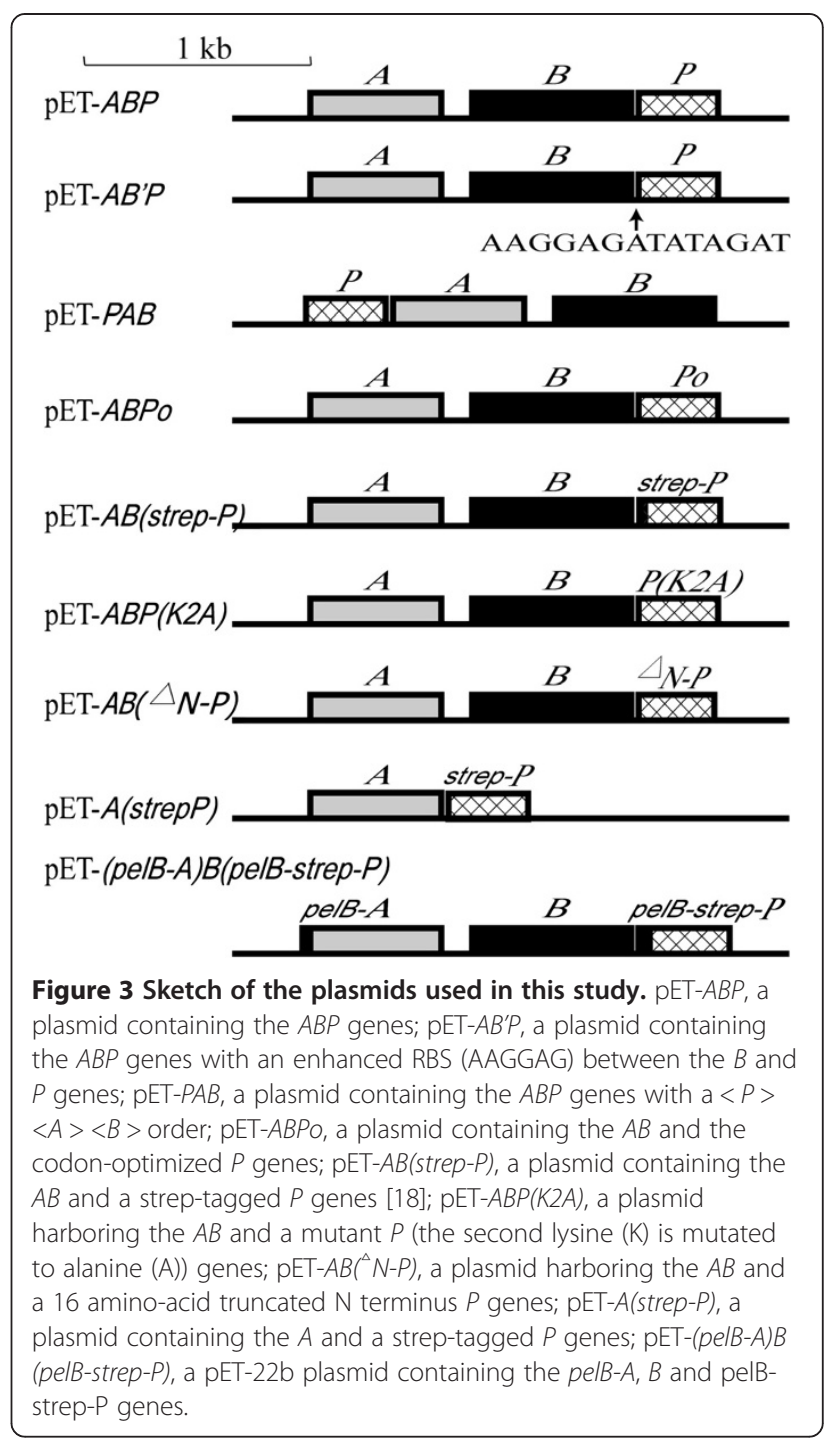

proteins [22,23] (Figure 1). We analyzed the N-terminal amino-acids of some weakly expressed NHase activators from Bordetella petrii DSM 12804, P. putida NRRL-18668, Bacillus BR449 and Bacillus RAPc8. Surprisingly, we found that all of the second N-terminal amino-acids of the NHase activators in these four strains are lysine (K) (Table 1). Thus, it may be that $\mathrm{N}$-end rule degradation leads to difficulty with the expression and isolation of P14K. Based on this speculation, we designed a mutant gene, $A B P(K 2 A)$, in which the Lys-2 in the N-terminus of P14K was substituted with $\mathrm{Ala}$ in the plasmid pET- $A B P(K 2 A)$. In addition, we analyzed the secondary structure of the $\mathrm{P} 14 \mathrm{~K}$ as predicted by JPRED3 (http://www.compbio.dundee.ac.uk/www-jpred/ index.html) and found the $\mathrm{N}$-terminal 16 amino-acid group is in an N-terminal loop (Figure 4B) and the 17th aminoacid is a Gly that could avoid P14K degradation effectively according the $\mathrm{N}$-end rule $[22,23]$. Therefore, we constructed plasmid pET- $A B\left({ }^{\wedge} N-P\right)$, in which the N-terminal 

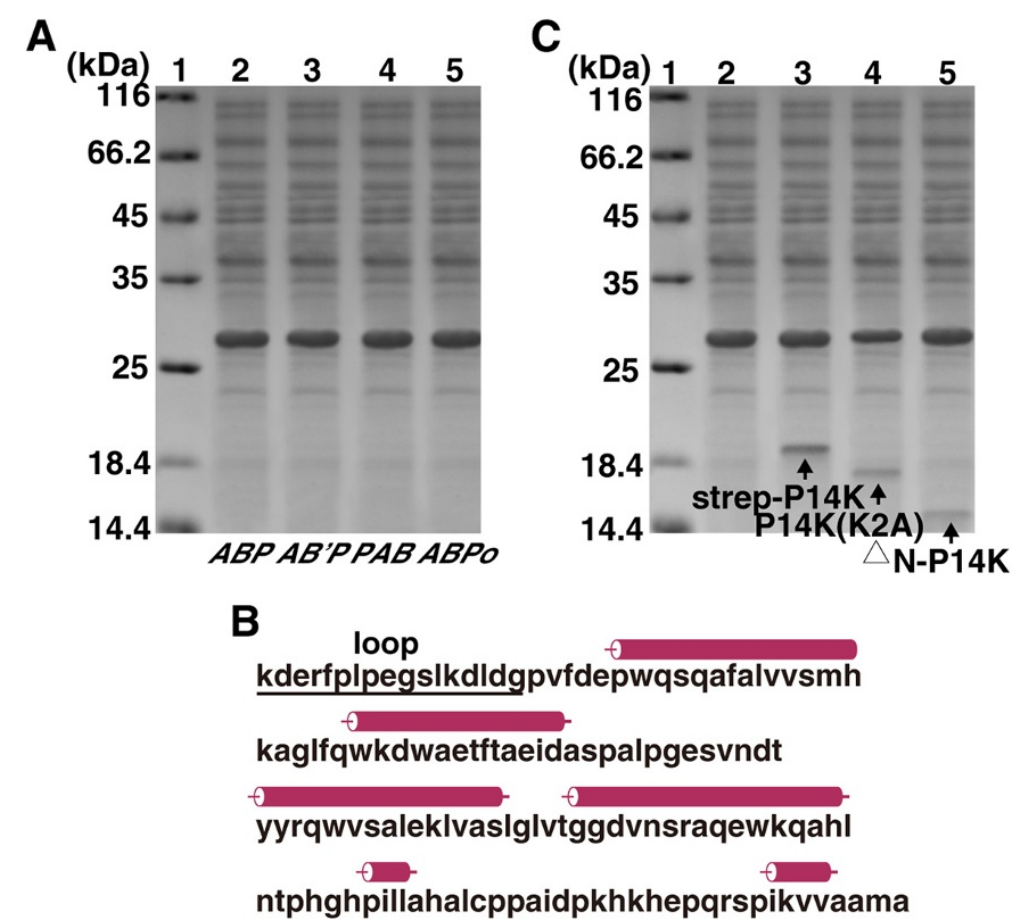

Figure 4 SDS-PAGE analysis of recombinant NHases and P14K, and the predicted secondary structure of P14K. (A) SDS-PAGE analysis of the cell extracts of the transformants carrying pET-ABP, pET-AB'P, pET-PAB or pET-ABPo. 1, mark; 2, pET-ABP; 3, pET-AB'P; 4, pET-PAB; 5, pET-ABPO. (B) Predicted secondary structure of P14K. (C) SDS-PAGE analysis of the cell extracts of the transformants carrying pET-AB(strep-P), pET-ABP(K2A) or $\mathrm{pET}-A B\left({ }^{\wedge} N-P\right)$. 1, mark; 2, pET-ABP; 3, pET-AB(strep-P); 4, pET-ABP(K2A); 5, harboring pET-AB( $\left.{ }^{\wedge} N-P\right)$.

16 residues of $\mathrm{P} 14 \mathrm{~K}$ were deleted, the second $\mathrm{N}$-terminal amino-acid in the truncated $P 14 \mathrm{~K}$ was Gly. The transformants harboring $\mathrm{pET}-A B P(K 2 A)$ and $\mathrm{pET}-A B\left({ }^{\wedge} N-P\right)$ were used for NHase and $\mathrm{P} 14 \mathrm{~K}$ expression. As shown in Figure $4 \mathrm{C}$, each mutant P14K was successfully expressed and the crude NHase activity in the cell-free extracts of each transformant was similar to that of the transformant harboring pET- $A B P(120.5 \mathrm{U} / \mathrm{mg})$. However, compared with the expression of $\mathrm{P} 14 \mathrm{~K}$ using the transformant harboring pET- $A B($ strep- $P$ ), the expression level of $\mathrm{P} 14 \mathrm{~K}$ in the two mutants was not improved (Figure 4C).

\section{High P14K expression yield}

It has been reported that exporting a protein to the periplasm to enhance its stability can be regarded as an effective strategy to optimize the production of recombinant

Table $1 \mathrm{~N}$-terminal amino-acid sequence of NHase activators in various strains

\begin{tabular}{ll}
\hline Strain & N-terminal amino-acid sequence \\
\hline Bordetella petrii DSM 12804 & M K DERLPLP (YP_001630019.1) \\
Pseudomonas putida NRRL-18668 & M K DERFPLP (P14K in this study*) \\
Bacillus BR449 & M K SCENQPN (AAF69003.1) \\
Bacillus RAPC8 & M K SCENQPN (AAS84452.1) \\
\hline
\end{tabular}

Protein accession numbers are shown in brackets. ${ }^{*}$ the correct $\mathrm{N}$-terminal amino-acid sequence of P14K was identified by our previous work [18]. proteins $[27,28]$. To yield large amounts of P14K in recombinant $E$. coli cells, we attempted to secrete P14K into the periplasmic space. As P14K formed a complex with the $\alpha$-subunit of NHase [18], we designed a mutant gene $($ pelB-A)B(pelB-strep- $P$ ) in which the pelB signal peptide was added upstream of the $A$ and strep- $P$ genes in the plasmid pET22b-(pelB-A)B(pelB-strep- $P)$. The transformant harboring $\mathrm{pET} 22 \mathrm{~b}-($ pelB-A$) B($ pelB-strep- $P)$ was used for recombinant $\mathrm{P} 14 \mathrm{~K}$ expression. Although no target protein was observed in the culture supernatant, a large amount of the full-length pelB-strep-P14K and pelB- $\alpha$ subunit (the pelB signal peptides were not cut off in either) were detected in the cell-free extract (Figure 5A). In addition, the enzyme activity in the cell-free extract was comparable to that of the wild-type NHase.

\section{Stability of pelB-strep-P14K}

To investigate the mechanism of how the fusion protein pelB-strep enhances the recombinant expression of P14K, we compared the difference in the protein stability between the purified P14K-containing activator complex [ $\alpha$-(strep$\mathrm{P} 14 \mathrm{~K})]$ and $[\alpha$-(pelB-strep-P14K)]. SDS-PAGE analysis was carried to investigate the stability of cobalt-containing $[\alpha-($ strep-P14K)] and $[\alpha-($ pelB-strep-P14K)] (a culture containing the cobalt ion) during storage at room temperature. As shown in Figure 5B, the pelB-strep-P14K band from the 

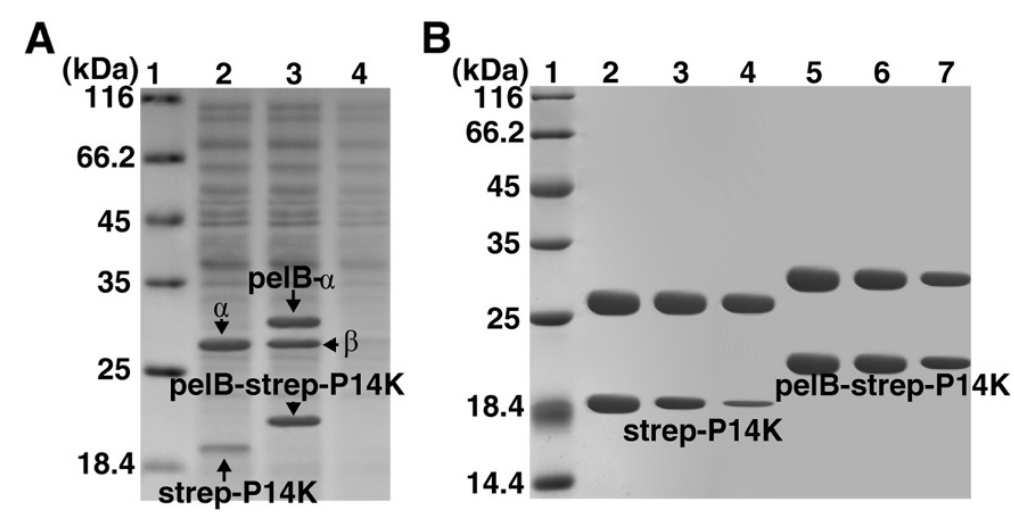

Figure 5 SDS-PAGE analysis of recombinant pelB-strep-P14K. (A) SDS-PAGE analysis of the activators encoded by $A($ strep-P) and (pelB-A)B (pelB-strep-P). 1, mark; 2 , cell extracts of the transformant containing $p E T-A(s t r e p-P)$; 3 , cell extracts of the transformant containing $p E T$ - $(p e l B-A) B$ (pelB-strep-P); 4, culture supernatant of the transformant containing pET-(pelB-A)B(pelB-strep-P). (B) SDS-PAGE analysis of stability of purified activator complexes [a-(strep-P14K)] and [a-(pelB-strep-P14K)]. 1, mark; 2, purified [a-(strep-P14K)]; 3, purified [a-(strep-P14K)] after storage for 1 day; 4, purified [a-(strep-P14K)] after storage for 2 days; 5, purified [a-(pelB-strep-P14K)]; 6, purified [a-(pelB-strep-P14K)] after storage for 2 days; 7, purified [a-(pelB-strep-P14K)] after storage for 6 days.

recombinant $[\alpha$-(pelB-strep-P14K)] complex decayed to $90 \%$ of the original intensity after 2 days of storage and eventually to $60 \%$ after 6 days. However, the strep-P14K band from the $[\alpha-($ strep-P14K)] complex decreased to $60 \%$ after only 1 day of storage and to $10 \%$ after 2 days. The finding that pelB-strep-P14K is far more stable than strepP14K indicated that thermal stability may be a key factor in P14K expression.

\section{Conclusions}

In conclusion, the activator P14K from P. putida NRRL18668 was successfully expressed based on the $\mathrm{N}$-end rule degradation, the stability of the P14K was improved by adding a pelB signal peptide. Further study of the influence of P14K on the maturation of NHase is currently underway. The strategy used for P14K expression in this study may be useful for the heterologous expression of other difficult expression proteins.

\section{Methods}

\section{Bacterial strain and plasmids}

NHase and the P14K gene $(A B P)$ were cloned from $P$. putida NRRL-18668. E. coli BL21 (DE3) was used as the host for the plasmid pET-24a(+), which was used for $A B P, A B^{\prime} P, P A B, A B P o, A B($ strep- $P), A B P(K 2 A), A B\left({ }^{\wedge} N-P\right)$ and $A($ strep- $P$ ) expression. The plasmid pET-22b was used for (pelB-A)B(pelB-strep-P) expression.

\section{Construction of plasmids}

The genomic DNA of $P$. putida NRRL-18668 was isolated for $A B P$ cloning with the primers $\mathrm{A}$-up and Pdown (Table 2 and Figure 3). The PCR products were digested and ligated into $\mathrm{pET}-24 \mathrm{a}(+)$ to generate the plasmid $\mathrm{pET}-A B P$. The recombinant plasmid $\mathrm{pET}-A B P$
Table 2 Oligonucleotide primers used in this study

\begin{tabular}{|c|c|}
\hline Name & Sequence \\
\hline A-up & 5'-GGAATTCCATATGGGGCAATCACACACGC-3' \\
\hline P-down & 5'-CCGGAATTCTCAAGCCATTGCGGCAACGA-3' \\
\hline B-down(rbs) & 5'-ATATCTATATCTCCTITCACGCTGGCTCCAGGTAGTC-3' \\
\hline P-up(rbs) & 5'-TGAAAGGAGATATAGATATGAAAGACGAACGGTTT-3' \\
\hline P-up & 5'-GGAATTCCATATGAAAGACGAACGGTTT-3' \\
\hline P-down(PAB) & 5'-CATATCTATATCTCCTTTCAAGCCATTGCGGCAACG-3' \\
\hline A-up(PAB) & 5'-TGAAAGGAGATATAGATATGGGGCAATCACACACGC-3' \\
\hline B-down(PAB) & 5'-CCGGAATTCTCACGCTGGCTCCAGGTAGT-3' \\
\hline Po-up & 5'-GGAATTCCATATGAAAGACGAACGTTC-3' \\
\hline Po-down & 5'-CCGGAATTCTCAAGCCATAGCAGCAACAA-3' \\
\hline B-down(strep) & $\begin{array}{l}\text { 5'-GCGGGTGGCTCCAGCTTGCCATATCTATATCTCCTITCA } \\
\text { CGCTGGCTCCAGGTAGTCATC-3' }\end{array}$ \\
\hline P-up(strep) & $\begin{array}{l}\text { 5'-AAGCTGGAGCCACCCGCAGTTCGAAAAGGGTGCAAAA } \\
\text { GACGAACGGTTTCCATT-3' }\end{array}$ \\
\hline K2A-up & 5'-TGAAAGGAGATATAGATATGGCGGACGAACGGTTTC-3' \\
\hline K2A-down & 5'-GCAATGGAAACCGTTCGTCCGCCATATCTATATCTCC-3' \\
\hline $\mathrm{P}-\mathrm{up}\left({ }^{\Delta} \mathrm{N}\right)$ & 5'-TGAAAGGAGATATAGATATGGGCCCTGTGTTTGACG-3' \\
\hline A-down(strep) & $\begin{array}{l}\text { 5'-GCGGGTGGCTCCAGCTTGCCATATCTATATCTCCTTTA } \\
\text { ATGAGATGGGGTGGGTTGGGT-3' }\end{array}$ \\
\hline secA-up & 5'-CCGGAATTCGGGGCAATCACACACGCAT-3' \\
\hline secB-down & $\begin{array}{l}\text { 5'-GATATCCATGGCCATCGCCGGCTGGGCAGCGAGGAG } \\
\text { CAGCAGACCAGCAGCAGCGGTCGGCAGCAGGTATTTCA } \\
\text { TATGTATATCTCCTITCACGCTGGC-3' }\end{array}$ \\
\hline secP-up & $\begin{array}{l}\text { 5'-CAGCCGGCGATGGCCATGGATATCGGAATTAATGCAA } \\
\text { GCTGGAGCCACCCGCAGTTCGAAAAGGGTGCAAAAGAC } \\
\text { GAACGGTTCCATTGCCAG-3' }\end{array}$ \\
\hline secP-down & 5'-CGACCCAAGCTTTCAAGCCATTGCGGCAACGACC-3' \\
\hline
\end{tabular}

Italicized letters denote the Ndel, EcoRI and Hindlll recognition sites, respectively. 
was then transformed into E. coli BL21 (DE3) for heterologous expression. An overlap extension PCR protocol was used for construction of the plasmid pET- $A B^{\prime} P$ ('represents an insertion of RBS sequence) through two rounds of PCR. The first round of PCR was performed using the primer pairs A-up and B-down(rbs) and P-up (rbs) and P-down, respectively, the plasmid pET-ABP was used as the template. The second round of PCR was performed to produce the full-length $A B^{\prime} P$, using the primers A-up and P-down and mixing equal molar amounts of the first-round products as template. The following plasmids were prepared similar to pET-AB'P. The plasmid pET-PAB was prepared with the primer pairs P-up and P-down(PAB) and A-up(PAB) and Bdown (PAB). The plasmid pET-ABPo was constructed with the primer pairs A-up and B-down(rbs) and Po-up and Po-down. The plasmid pET-Po (Po, codon optimized $P$ gene synthesized by Sangon Biotech Ltd.) was used as the template. The plasmid pET- $A B($ strep- $P$ ) was constructed with the primer pairs A-up and B-down (strep) and P-up(strep) and P-down as described previously [18]. The plasmid pET- $A B\left({ }^{\wedge} N-P\right)$ was constructed with the primers A-up and B-down(rbs) and $\mathrm{P}$-up $\left({ }^{\triangle} \mathrm{N}\right)$ and P-down. The plasmid pET-A(strep- $P$ ) was generated from pET- $A B P$ with the primers A-up and A-down (strep) and P-up(strep) and P-down. The plasmid pET22b-(pelB-A)B(pelB-strep-P) was prepared with the primers of secA-up and secB-down and secP-up and P-down. The plasmid pET- $A B P(K 2 A)$ mutant was constructed to produce full-length $A B P(K 2 A)$ with the primers K2A-up and K2A-down using pET- $A B^{\prime} P$ as the template. The PCR products were digested with DpnI to degrade the template plasmid and then transformed into E. coli BL21 (DE3).

Expression and purification of enzymes and enzyme assay E. coli BL21 (DE3) transformants containing the recombinant plasmids were grown at $37^{\circ} \mathrm{C}$ in $\mathrm{TB}$ medium containing $\mathrm{CoCl}_{2} \cdot 6 \mathrm{H}_{2} \mathrm{O}(0.05 \mathrm{~g} / \mathrm{l})$ and kanamycin $(50 \mu \mathrm{g} /$ $\mathrm{ml})$ until culture $A_{600}$ reached 0.8 . Isopropyl $\beta$-D-thiogalactopyranoside was added to a final concentration of $0.4 \mathrm{mM}$. The cells were then incubated at $24^{\circ} \mathrm{C}$ for $16 \mathrm{~h}$.

All purification steps were performed at $4^{\circ} \mathrm{C}$. The procedures were conducted with an AKTA purifier (GE Healthcare UK Ltd.). Potassium phosphate buffer (KPB) (10 mM, pH 7.5) containing $0.5 \mathrm{mM}$ dithiothreitol (DTT) was used in the purification steps. Both NHase and its activator complex were purified with a HisTrap HP column (GE Healthcare UK Ltd.). The target proteins were eluted off the column with gradient concentrations of imidazole from $0 \mathrm{mM}$ to $500 \mathrm{mM}$ (40 mM, $80 \mathrm{mM}, 200 \mathrm{mM}, 300 \mathrm{mM}$ and $500 \mathrm{mM})$ in $10 \mathrm{mM}$ KPB. The preliminarily separated proteins were further purified with a Hiload 16/60 Superdex 200 pg column
(GE Healthcare UK Ltd.). The process of separation and purification was monitored by SDS-PAGE analysis.

NHase activity was assayed in a reaction mixture comprising $10 \mathrm{mM} \mathrm{KPB} \mathrm{(pH} \mathrm{7.5),} 20 \mathrm{mM}$ 3-cyanopyridine as a substrate and $0.1 \mu \mathrm{g}$ enzyme in a total volume of $500 \mu \mathrm{L}$. The reaction mixture was incubated at $20^{\circ} \mathrm{C}$ for $20 \mathrm{~min}$ and terminated by addition of $500 \mu \mathrm{L}$ of acetonitrile. The activity of NHase was determined by monitoring the formation of nicotinamide in the reaction mixture with high-pressure liquid chromatography (HPLC) as previously described [13]. One unit of NHase activity was defined as the amount of enzyme that catalyzed the formation of $1 \mu \mathrm{mol}$ of nicotinamide per min at $20^{\circ} \mathrm{C}$.

\section{Competing interests}

The authors declare that there are no competing interests.

\section{Authors' contributions}

ZMZ, WJC and MK designed this study. YL, YQF, YCY, YTC and YYX performed the experimental work. ZMZ, WJC, YL and MK wrote the manuscript. ZMZ and MK collaborated in the coordination of the research and helped to draft the manuscript. All authors read and approved the submission of the manuscript.

\section{Acknowledgements}

This work was supported by the National Natural Science Foundation of China (31070711), the New Century Excellent Talents in University (NCET-100461), Fundamental Research Funds JUSRP20909 from the Central Universities 111 Project (111-2-06), the Doctoral Scientific Research Fund Project of Jiangnan University of China (JUDCF10011), the General University Doctor Research and Innovation Program of Jiangsu Province of China (CXZZ11_0475).

\section{Author details}

${ }^{1}$ Key Laboratory of Industrial Biotechnology, Ministry of Education, School of Biotechnology, Jiangnan University, Wuxi 214122, Peoples Republic of China. ${ }^{2}$ School of Environmental and Civil Engineering, Jiangnan University, Wuxi 214122, Peoples Republic of China. ${ }^{3}$ Institute of Applied Biochemistry, and Graduate School of Life and Environmental Sciences, The University of Tsukuba, 1-1-1 Tennodai, Tsukuba, Ibaraki 305-8572, Japan.

Received: 30 January 2013 Accepted: 30 May 2013

Published: 3 June 2013

\section{References}

1. Noguchi T, Nojiri M, Takei K, Odaka M, Kamiya N: Protonation structures of Cys-sulfinic and Cys-sulfenic acids in the photosensitive nitrile hydratase revealed by Fourier transform infrared spectroscopy. Biochemistry 2003, 42:11642-11650.

2. Kobayashi M, Shimizu S: Cobalt proteins. Eur J Biochem 1999, 261:1-9.

3. Kobayashi M, Fujiwara Y, Goda M, Komeda H, Shimizu S: Identification of active sites in amidase: evolutionary relationship between amide bondand peptide bond-cleaving enzymes. Proc Natl Acad Sci U S A 1997, 94:11986-11991.

4. Nagashima S, Nakasako M, Dohmae N, Tsujimura M, Takio K, Odaka M, Yohda M, Kamiya N, Endo I: Novel non-heme iron center of nitrile hydratase with a claw setting of oxygen atoms. Nat Struct Mol Biol 1998, 5:347-351.

5. Murakami T, Nojiri M, Nakayama H, Dohmae N, Takio K, Odaka M, Endo I, Nagamune T, Yohda M: Post-translational modification is essential for catalytic activity of nitrile hydratase. Protein Sci 2000, 9:1024-1030.

6. Miyanaga A, Fushinobu S, Ito K, Shoun H, Wakagi T: Mutational and structural analysis of cobalt-containing nitrile hydratase on substrate and metal binding. Eur J Biochem 2003, 271:429-438.

7. Arakawa T, Kawano Y, Kataoka S, Katayama Y, Kamiya N, Yohda M, Odaka M: Structure of thiocyanate hydrolase: a new nitrile hydratase family 
protein with a novel five-coordinate cobalt (III) center. J Mol Biol 2007 366:1497-1509.

8. Okamoto S, Eltis LD: The biological occurrence and trafficking of cobalt. Metallomics 2011, 3:963-970.

9. Nojiri M, Yohda M, Odaka M, Matsushita Y, Tsujimura M, Yoshida T, Dohmae $\mathrm{N}$, Takio K, Endo I: Functional expression of nitrile hydratase in Escherichia coli: requirement of a nitrile hydratase activator and post-translational modification of a ligand cysteine. J Biochem 1999, 125:696-704.

10. Nishiyama M, Horinouchi S, Kobayashi M, Nagasawa T, Yamada H, Beppu T: Cloning and characterization of genes responsible for metabolism of nitrile compounds from Pseudomonas chlororaphis B23. J Bacteriol 1991, 173:2465-2472.

11. Hashimoto Y, Nishiyama M, Horinouchi S, Beppu T: Nitrile hydratase gene from Rhodococcus sp. $\mathrm{N}-774$ requirement for its downstream region for efficient expression. Biosci Biotechnol Biochem 1994, 58:1859-1865.

12. Lu J, Zheng Y, Yamagishi H, Odaka M, Tsujimura M, Maeda M, Endo I: Motif CXCC in nitrile hydratase activator is critical for NHase biogenesis in vivo. FEBS Lett 2003, 553:391-396.

13. Zhou Z, Hashimoto Y, Shiraki K, Kobayashi M: Discovery of posttranslational maturation by self-subunit swapping. Proc Natl Acad Sci U S A 2008, 105:14849-14854.

14. Zhou ZM, Hashimoto Y, Cui TW, Washizawa Y, Mino H, Kobayashi M: Unique Biogenesis of High-Molecular Mass Multimeric Metalloenzyme Nitrile Hydratase: Intermediates and a Proposed Mechanism for SelfSubunit Swapping Maturation. Biochemistry 2010, 49:9638-9648.

15. Zhou ZM, Hashimoto Y, Kobayashi M: Self-subunit Swapping Chaperone Needed for the Maturation of Multimeric Metalloenzyme Nitrile Hydratase by a Subunit Exchange Mechanism Also Carries Out the Oxidation of the Metal Ligand Cysteine Residues and Insertion of Cobalt. J Biol Chem 2009, 284:14930-14938.

16. Okamoto S, Van Petegem F, Patrauchan MA, Eltis LD: AnhE, a metallochaperone involved in the maturation of a cobalt-dependent nitrile hydratase. J Biol Chem 2010, 285:25126-25133.

17. Wu S, Fallon RD, Payne MS: Over-production of stereoselective nitrile hydratase from Pseudomonas putida 5B in Escherichia coli: activity requires a novel downstream protein. Appl Microbiol Biotechnol 1997, 48:704-708.

18. Liu Y, Cui W, Xia Y, Cui Y, Kobayashi M, Zhou Z: Self-subunit swapping occurs in another gene type of cobalt nitrile hydratase. PLoS One 2012, 7:e50829.

19. Varshavsky A: The $\mathrm{N}$-end rule pathway of protein degradation. Genes Cells 1997, 2:13-28

20. Mogk A, Schmidt R, Bukau B: The N-end rule pathway for regulated proteolysis: prokaryotic and eukaryotic strategies. Trends Cell Biol 2007, 17:165-172.

21. Dougan $\mathrm{D}$, Truscott $\mathrm{K}$, Zeth $\mathrm{K}$ : The bacterial $\mathrm{N}$-end rule pathway: expect the unexpected. Mol Microbiol 2010, 76:545-558.

22. Tobias JW, Shrader TE, Rocap G, Varshavsky A: The N-end rule in bacteria. Science 1991, 254:1374-1377.

23. Dougan D, Micevski D, Truscott K: The $\mathrm{N}$-end rule pathway: From recognition by N-recognins, to destruction by AAA + proteases. Biochim Biophys Acta 1823, 2012:83-91.

24. Salis HM, Mirsky EA, Voigt CA: Automated design of synthetic ribosome binding sites to control protein expression. Nat Biotechnol 2009, 27:946-950.

25. Icev A, Ruiz C, Ryder EF: Distance-enhanced association rules for gene expression. Gene 2003, 10:34-40.

26. Sahdev S, Khattar SK, Saini KS: Production of active eukaryotic proteins through bacterial expression systems: a review of the existing biotechnology strategies. Mol Cell Biochem 2008, 307:249-264.

27. Kwon WS, Da Silva NA, Kellis JT Jr: Relationship between thermal stability, degradation rate and expression yield of barnase variants in the periplasm of Escherichia coli. Protein Eng 1996, 9:1197-1202.

28. Choi J, Lee S: Secretory and extracellular production of recombinant proteins using Escherichia coli. Appl Microbiol Biotechnol 2004, 64:625-635.

doi:10.1186/1472-6750-13-48

Cite this article as: Liu et al.: Strategy for successful expression of the Pseudomonas putida nitrile hydratase activator P14K in Escherichia coli. BMC Biotechnology 2013 13:48.

\section{Submit your next manuscript to BioMed Central and take full advantage of:}

- Convenient online submission

- Thorough peer review

- No space constraints or color figure charges

- Immediate publication on acceptance

- Inclusion in PubMed, CAS, Scopus and Google Scholar

- Research which is freely available for redistribution

Submit your manuscript at www.biomedcentral.com/submit
Ciomed Central 